\title{
La transformación de las democracias \\ latinoamericanas: el replanteamiento de \\ la relación Estado-sociedad. Caso Brasil
}

\author{
The Transformation of Latin American \\ Democracies: Reassessment of the State-society \\ Relationship: The Brazilian Case.
}

\section{Ilana Aló Cardoso Ribeiro}

Recepción: 28 de agosto de 2015

Aceptación: 4 de septiembre de 2015

\section{Resumen}

Este artículo tiene como objetivo abordar las transformaciones de la democracia brasileña desde el final de la dictadura militar en 1985, pasando por los gobiernos neoliberales de derecha $(\mathrm{PSDB})^{1}$ hasta su giro a la izquierda $(\mathrm{PT})^{2}$ para entender la relación Estadosociedad. A través de un breve recuento histórico, que no pretende ser exhaustivo sino ilustrativo, busca contestar la pregunta clave de esa investigación: ¿¿desde el retorno a la democracia hubo un replanteamiento de la relación Estado-sociedad? Para ello, abordaremos además el papel que juega el sistema de partidos en Brasil. El artículo se basa en dos ejes: el primero ilustra el retorno a la democracia y los primeros gobiernos de derecha neoliberales y el segundo busca analizar los gobiernos de izquierda, ambos ligados al eje central que es la relación Estado-sociedad. La metodología utilizada es un análisis histórico/político a partir de fuentes como publicaciones periodísticas y bases bibliográficas.

Palabras clave: Brasil, democracia, transformaciones, Estado, sociedad.

\section{Abstract}

The goal of this article is to address the transformations of the Brazilian democracy since the end of the military dictatorship in 1985, passing through neoliberal right-wing governments (PSDB) until its turn towards the left-wing government (PT), in order to understand the State-society relationship. Through a brief historical review, which is not intended to be exhaustive yet illustrative, it looks for answering the key question of this research: has there been a reassessment of the relationship State-society since the return to

1 PSDB - Partido da Social Democracia Brasileira http://www.psdb.org.br/

2 PT - Partido dos Trabalhadores http://www.pt.org.br/ 
democracy? Consequently, the role of the Brazilian parties system will be also addressed. Therefore, this work is based upon two central concepts: the first one illustrates the return to democracy as well as the first neoliberal right-wing governments; and the second one analyses the left-wing governments; both of them are linked to a central concept, which is the State-society relationship. The methodology used is a historical and political analysis of sources such as journalist publications and bibliographical data-basis.

Keywords: Brazil, democracy, transformations, State, society. 


\section{Introducción}

1 objetivo de ese artículo es abordar las transformaciones de la democracia brasileña para verificar si hubo un replanteamiento en la relación Estado-sociedad. Y es observando la República Federativa de Brasil desde su primera o vieja República (1890-1930), pasando por la dictadura del Estado Nuevo con Getulio Vargas (1930-1945), su época de expansión en la República Nueva (1946-1964), la dictadura militar (1964-1985) hasta el retorno a la democracia (1985), que se puede analizar esa relación.

Partiendo de la pregunta de investigación propuesta, se indaga si ¿desde el retorno a la democracia hubo un replanteamiento de la relación Estado-sociedad? Y para contestar esa pregunta es importante destacar aspectos teóricos, históricos y políticos en el escenario brasileño.

Antes, vale la pena observar que un estudio profundizado sobre el tema debe contener muchos elementos que quizás quede corto para un único ensayo, por eso se debe considerar una expansión en futuros estudios. Además, el presente trabajo no pretende agotar el tema sino abordarlo de manera que se pueda entender las transformaciones de la democracia brasileńa a partir de una metodología basada en un análisis histórico y político.

Para tanto serán utilizados dos ejes ligados al eje central que es el replanteamiento o no de la relación Estado-sociedad: el primer punto ilustra el retorno a la democracia, la crisis y los primeros gobiernos de derecha neoliberales; el segundo busca analizar los gobiernos de izquierda, su nuevo modelo social y el retorno a la crisis.

La estructura del trabajo está compuesta por: (1) un aporte histórico donde se encuentra un breve recuento de la historia política brasileña desde el inicio de la República; (2) un aporte teórico que aborda las teorías sobre la redemocratización de Latinoamérica, el neoliberalismo y el socialismo del siglo XXI; (3) el primer eje, que aborda el retorno a la democracia, el gobierno de derecha, crisis y políticas neoliberales; (4) el 
segundo eje que es sobre el giro a la izquierda, un nuevo modelo social y el retorno a la crisis; y (5) algunas consideraciones finales.

Como metodología se optó por hacer un análisis histórico/político basado en periódicos brasileños, seleccionados por su historia, ya que se relacionan desde los inicios de la República hasta los días actuales. También se hizo una revisión bibliográfica que da el soporte teórico necesario.

\section{Aporte histórico: Un recuento de la historia política brasileña republicana}

No muy diferente de la historia latinoamericana, Brasil es parte de un contexto de inestabilidad política y social marcado por regímenes antidemocráticos, dictaduras y golpes a lo largo de los últimos siglos. Eso se puede observar desde la raíz de la República que, fundada el 15 de noviembre de 1889, es fuente de un golpe militar liderado por el General Deodoro da Fonseca.

Desde entonces Brasil tuvo una primera República, también llamada República Vieja, que va de 1890 hasta 1930. En esa época el contexto era de un país de estructura oligárquica que giraba alrededor de dos Estados productores de café: Minas Gerais y São Paulo, que eran los polos dinámicos de la economía nacional ${ }^{3}$.

Con la crisis política originada por el fin del pacto oligárquico, en las elecciones de 1930 asume el presidente Washington Luis que es depuesto por una junta gubernamental. Se da paso a un gobierno provisional donde asume el poder Getulio Vargas.

Post revolución de 1932, en 1934 se aprueba una nueva Constitución donde el gobierno constitucional ratifica a Getulio Vargas en el poder, iniciando así el Estado nuevo o la Era Vargas, la primera dictadura brasileña donde Vargas se mantiene en el poder desde 1930 hasta

3 Vale recordar que estamos tratando de un país federal por eso hablamos de una estructura de Estados federales y de un Gobierno Central Federal que es denominado de Unión. 
LA TRANSFORMACIÓN DE LAS DEMOCRACIAS LATINOAMERICANAS: EL REPLANTEAMIENTO DE LA RELACión Estado-SOciedad. Caso Brasil

\section{5.}

Considerado el primer líder populista a pesar de su dictadura, Vargas busca la unificación de una identidad nacional (Ruiz-Peinado, 2003). Esa unificación lo hace a través de una política social económica de introducción de derechos laborales y de la intervención del trabajo en la economía (industrialización, creación de la VALE ${ }^{4}$ y de la PETROBRAS), aunque restringiendo otros derechos como las libertades individuales y colectivas.

Se puede observar la relación Estado-sociedad cuando se levanta un líder populista. Aunque en las ciencias sociales populismo es frecuentemente utilizado como forma de gobierno, este término no dispone de una formulación teórica precisa y acabada. En general, tiene una connotación negativa ligada a coyunturas políticas donde los gobernantes y políticos son considerados grandes demagogos y a veces manipuladores populares, de ahí que el término actúe como un significante flotante y vacío ${ }^{5}$.

Así, la centralidad de la figura del líder en el populismo lleva a una identificación de la unidad del grupo, el líder es aquel que puede venir a asumir la función de significación -la unificación simbólica del grupo alrededor de una individualidad es inherente a la formación de un pueblo (pueblo visto como una categoría política) ${ }^{7}$-. El populismo enton-

4 VALE es una empresa de extracción de minerales como hierro y níquel.

http://www.vale.com/brasil/PT/Paginas/default.aspx

Petrobras es la petrolera estatal del país. http://www.petrobras.com/en/home.htm

5 Cancian, Renato. Populismo: Fenômeno político baseia-se no carisma de gobernantes.

http://www.vale.com/brasil/PT/Paginas/default.aspx

6 De acuerdo con esa visión, un tanto marxista, las masas, o clases populares, son consideradas amorfas por no haber adquirido consciencia de clase y por eso se vuelve susceptible a la dominación retórica de los que pretenden conquistar o mantener el poder. Según Carlos de la Torre "el populismo, de acuerdo a esta visión, se basaría en la apropiación autoritaria de la voluntad popular por un líder autoerigido y visto por sus seguidores como la condensación de las demandas de ruptura y las promesas de redención". Entretanto, Laclau articula el populismo, con campos del psicoanálisis, de lingüística y retórica para demostrar que el populismo no posee una única unidad referencial y es considerado una forma de construir lo político que está directamente ligada a demanda(s) democrática(s) y demanda(s) popular(es). Para él, el populismo debería ser rescatado de su posición marginal del discurso de las ciencias sociales por ser considerado una dimensión constante de la acción política, aunque actúe como significante flotante y vacío.

7 Laclau, Ernesto. A Razẫo Populista. Rio de Janeiro: EdUERJ, 2013. 
ces apoyado en la figura de un líder carismático que tiene una relación de proximidad con el pueblo, en determinados contextos históricos, logra su influencia permanente.

Es el caso de Getulio Vargas, que a pesar de haber sido electo presidente de forma indirecta y ser el protagonista de una dictadura, se vuelve un líder de características populistas creando así una relación ambigua de concesión y privación de derechos. Con ese liderazgo la relación existente entre el Estado y la sociedad también se vuelve ambigua pues hay apoyo al líder y a la vez rechazo.

Con el fin de la segunda guerra mundial en 1945, el entonces presidente Getulio Vargas es presionado a renunciar, ministros militares asumen el poder poniendo fin al Estado Nuevo. Empieza un periodo conocido por República Populista, República Nueva, República de 46 o Cuarta República Brasileña que va desde 1946 hasta 1964, donde ocurrieron elecciones directas, pero en el país aún había una fuerte influencia militar en el congreso, senado y ministerios.

La asamblea constituyente de 1946 elije a Eurico Gaspar Dutra como presidente, y este permanece en el poder hasta 1951 cuando regresa a la presidencia, esta vez por voto popular, Getulio Vargas. Con su suicidio en 1954, por razones políticas, asume su vicepresidente, Café Filho.

Hasta el golpe militar de 1964, dos presidentes más fueron electos a través del voto popular: Juscelino Kubitschek (1956-1961), el responsable por la construcción de la capital federal Brasilia y Janio Quadros (Enero 1961- agosto 1961) que renunció poco tiempo después de su posesión, asumiendo su vicepresidente João Goulart (1961-1964) ${ }^{8}$.

En 1964 empieza el régimen militar que se extendió hasta el retorno de la democracia en 1985. Lo destacable de esta etapa son los actos institucionales (AI) que cada vez más restringían las libertades individuales y colectivas a la vez que permitían al gobierno militar editar normas y decretos que estaban por encima de la constitución.

8 En ese recuento no están especificados los presidentes interinos porque esos se quedaron en el poder por cortos espacios de tiempo. Fuente: http://www.biblioteca.presidencia.gov.br/ex-presidentes. 
Fueron 17 actos institucionales emitidos por el gobierno militar, pero el AI 5 (1968) fue considerado el más autoritario, pues, en sus doce artículos concedía al presidente de la República plenos poderes. Entre ellos estaba el poder de suspender mandatos y derechos políticos de cualquier persona, intervenir en Estados y municipios, decretar receso en el congreso y suspender habeas corpus para crímenes políticos, además de permitir la censura irrestricta de todos los medios de comunicación?.

No había una relación pacífica entre la sociedad y el Estado, esa relación fue marcada por conflictos y restricciones hasta explotar en manifestaciones populares que ganaron fuerza con el debilitamiento del régimen militar, que con la tercera ola democrática en Latinoamérica llegó a su fin (1985).

Pero, aunque el régimen militar estuvo marcado por una gran represión, es importante recalcar que también estuvo marcado por el crecimiento económico con la expansión petrolera, la modernización del país y por inversiones en las industrias de base impulsadas por una campaña de nacionalización.

Su fin llega por una gran presión social estimulada por la campańa de "directas ya" que lleva a cabo la elección, aunque indirecta, del primer presidente civil después del largo periodo de dictadura: Tancredo Neves ${ }^{10}$. El presidente electo no logró asumir la presidencia a causa de una grave enfermedad y fue sustituido por José Sarney que permaneció en el cargo hasta la primera elección directa con sufragio universal en 1990 donde se eligió a Fernando Collor de Mello.

El regreso a la democracia en Brasil entonces, es marcado por la car-

9 El texto integral del AI5 es encontrado en la página web del Senado Federal http://legis.senado. gov.br/legislacao/ListaPublicacoes. action?id=194620

Mas informaciones sobre el tema se puede encontrar en los enlaces periodísticos que siguen: http://www.jblog.com.br/hojenahistoria.php?itemid=6283 http://www1.folha.uol.com.br/folha/treinamento/hotsites/ai5/

10 "Diretas já" fue un movimiento popular de la década de los 80’s que exigía el fin de la dictadura militar y elecciones directas. El PMDB fue el partido de oposición al régimen militar más actuante en ese movimiento. Fuentes: http://www1.folha.uol.com.br/especial/2014/30anosdasdiretasja/ ; http://memoriaglobo.globo.com/erros/diretas-ja.htm 
ta constitucional de 1988 que, entre otras cosas, devuelve las garantías y derechos fundamentales e irrestrictos suspendidos en el periodo de dictadura militar, además de promover elecciones populares y universales en 1990. Se inicia el periodo de consolidación de la democracia que es marcado por una gran crisis política y económica, corrupción y derrocamiento del primer presidente como veremos más adelante.

Los hechos abren espacio para un nuevo gobierno de derecha que tiene como principal característica la implementación de las políticas neoliberales del consenso de Washington y la implementación de un Estado mínimo con Fernando Henrique Cardoso y el PSDB. La historia va a culminar con el retorno de un Estado garantista y la búsqueda de la igualdad social, lo que se entiende como el socialismo del siglo XXI a través de la izquierda de Luis Ignacio Lula da Silva y Dilma Rousseff, ambos del PT.

En este punto, para que se pueda dar continuidad a la historia y entender la relación Estado-sociedad, se hace necesario un aporte teórico para especificar los términos que serán empleados en este artículo.

\section{Aporte teórico: Las teorías sobre la redemocratización de Latinoamérica, neoliberalismo y socialismo del siglo XXI}

Para hablar de redemocratización o transiciones hacia la democracia es necesario primero identificar lo que se entiende por democracia. Para tanto, existen autores claves que trabajan el tema como Giovanni Sartori (1987), Samuel P. Huntington (1989), Joseph A. Schumpeter (1947), Adam Przeworski (1998), Norberto Bobbio (2013), Alain Touraine (1995) Robert A. Dahl (1989), entre otros.

Es importante destacar que algunos países de Europa, así como Latinoamérica, son parte de la tercera ola de democratización.

"Una ola de democratización es un conjunto de transiciones de un régimen no democrático a otro democrático, que ocurren en determi- 
nado periodo de tiempo y que superan significativamente a las transiciones en dirección opuesta durante ese mismo periodo" (Huntington, 1994:26).

Fue en la década del 70 que países como Portugal, Espańa y Grecia iniciaron sus transiciones hacia la democracia, cuando empezaron a estructurar sus regímenes políticos. Ese modelo es replicado en toda Europa, "si se pone a un lado el caso especial de Alemania del Este, se suman 32 países que en el curso de las últimas décadas fueron autocracias y ahora parecen embarcar en ese tipo de transiciones" (Nun, 1995:30).

Según Samuel P. Huntington, existieron en el mundo moderno tres olas de democratización. La tercera, que es la que corresponde a América Latina, tiene sus inicios en la década de los 70's con Ecuador y la salida de los militares en 1977, la promulgación de una nueva constitución en 1978 y la elección de un presidente civil en 1979. El próximo país es Bolivia con la elección de un presidente civil en 1982. Luego Argentina con elecciones en 1983, Uruguay en 1984, siguiéndoles otros países (Huntington, 1994).

Como estamos tratando de la transición hacia la democracia, para fines de ese artículo es pertinente adoptar la definición mínima de democracia que defienden Huntington y Schumpeter basada en elecciones agregando el principio de la alternabilidad de Przeworski.

Para Samuel de P. Huntington el concepto de democracia tiene una connotación histórica ya que después de la Segunda Guerra Mundial ese concepto necesitaba de consolidación. En esa ola de redemocratización de los países de la posguerra, cada uno utilizaba el concepto de democracia como le parecía más conveniente según sus propios intereses, proliferando las democracias con adjetivos: democracia liberal o burguesa, democracia representativa, democracia directa, democracia totalitaria.

Por eso crea el concepto de democracia que se define como mínimo. O sea, el concepto mínimo de democracia consiste básicamente en que las personas que toman las decisiones por el Estado sean electas a través de elecciones limpias, honestas y periódicas, con base en el sufragio 
universal, con participación plena y libertades civiles.

El centro de la teoría de Schumpeter es concebir la democracia como un método, es decir, como un modo de proceder cuya ausencia o inexistencia se vuelve fácil de comprobar en la mayoría de los casos. Este concepto viene de una base empírica que se sujeta a los hechos e instituciones que permiten o no su funcionamiento que es formado por una concepción abstracta de componentes ideales.

No es considerada una definición que pretende ser exhaustiva sino que garantice un método para conseguir decisiones formalmente por medios democráticos, considerada una definición también minimalista que se basa en elecciones, coincidiendo en ese punto con Huntington. Así, define que

"democracia es un método político, un cierto arreglo institucional para llegar a decisiones político-legislativas y administrativas. Ese método democrático es un instrumento institucional para llegar a decisiones políticas, en el cual cada individuo tiene el poder de decidir a través del voto popular" (Schumpeter, 1947).

En una visión más moderna y en la misma vertiente de los autores anteriores, el concepto de democracia para Adam Przeworski trae las elecciones como un componente esencial. El autor establece el concepto de democracia electoral, y añade a las elecciones periódicas, libres y basadas en el sufragio universal, la alternabilidad en el poder como un principio básico. "Esto es lo distintivo de las democracias: los gobernantes son seleccionados mediante elecciones” (Przeworski, 1998).

En resumen, un sistema político es democrático siempre que "la mayoría de los que toman decisiones colectivas del poder sean seleccionados a través de limpias, honestas y periódicas elecciones, en las que los candidatos compiten libremente por los votos y en las que virtualmente toda población adulta tiene derecho a votar. Definida así la democracia abarca las dos dimensiones - competencia y participación" (Huntington, 1994:20). 
Definida la cuestión democrática, otro término utilizado en el trabajo que carece de base teórica es el neoliberalismo. Políticas neoliberales fueron empleadas en Latinoamérica a partir de los 80's a través de la aplicación de las directrices del Consenso de Washington ${ }^{11}$. "Estas medidas, en general, impulsaban la liberación del comercio y la no restricción de las importaciones, registraban una tendencia a la privatización de las empresas estatales y una desregulación a cualquier medida que pudiese obstaculizar el crecimiento del mercado. Todas ellas contienen una lógica de fomentar el comercio y la economía, que traspasa cualquier trazado de fronteras o derechos" (Montahuano, 2015).

Aquí, utilizaremos la definición de David Harvey que ejemplifica que

"el neoliberalismo es, ante todo, una teoría de prácticas político-económicas que afirma que la mejor manera de promover el bienestar del ser humano consiste en no restringir el libre desarrollo de las capacidades y de las libertades empresariales del individuo dentro de un marco institucional caracterizado por derechos de propiedad privada fuertes, mercados libres y libertad de comercio. El papel del Estado es crear y preservar el marco institucional apropiado para el desarrollo de éstas prácticas" (Harvey 2013: 6).

La próxima cuestión está ligada a lo que algunos autores llaman socialismo del siglo XXI o el retorno del Estado. El socialismo del siglo XXI es un concepto creado inicialmente por el sociólogo Heinz Dieterich Steffan y difundido por el presidente venezolano Hugo Chávez durante el V Foro Social Mundial en el 2005. Consiste básicamente en una mayor y más efectiva presencia estatal a través de políticas direccionadas a disminuir las desigualdades sociales.

Se desarrolla en un contexto donde el Estado pretende reaparecer e implementar políticas post-neoliberales. Tiene como característica principal un giro a la izquierda que marca una nueva forma de hacer política en algunos países de Latinoamérica como por ejemplo Argenti-

11 Se puede consultar el texto en inglés en la página de Harvard University. http://www.cid.harvard.edu/cidtrade/issues/washington.html 
na, Bolivia, Brasil, Ecuador y Venezuela.

Con los conceptos claves amparados teóricamente es posible trazar un panorama de la política brasileńa desde su retorno a la democracia hasta los días actuales. El periodo histórico que vamos a tratar se refiere a las primeras elecciones en 1990 con Collor de Melo, pasando por los gobiernos de derecha del PSDB culminando en los gobiernos de izquierda del PT. El sistema de partidos en ese punto es importante para entender la relación Estado-sociedad que se va forjando.

\section{Gobierno de derecha, crisis y políticas neoliberales}

Brasil es un país de sistema presidencialista. Sistema que fue elegido en la Constitución de $1988^{12}$ y luego ratificado en una consulta popular realizada en 1993 a fin de consultar a la población sobre el tipo de sistema de gobierno a ser adoptado en el país (monarquía, parlamentarismo o presidencialismo). Como ya se ha dicho, el país venía de una dictadura militar que duró veintiún ańos (1965-1985), fue gobernado por José Sarney (presidente interino) hasta que se realizaron elecciones directas donde triunfó Fernando Collor de Mello, el presidente más joven de la historia del país, con entonces cuarenta años ${ }^{13}$.

Collor empieza su mandato el día 15 de marzo de 1990 después de derrotar al candidato Luiz Inácio Lula da Silva en segunda vuelta. Pero, su gobierno fue perdiendo paulatinamente sustentación política, marcado por escándalos de corrupción y medidas administrativas impopulares. Entre esas medidas se destacan medidas radicales de contención de la inflación y estabilización de la moneda, privatización de empresas estatales, retirada de subsidios de exportaciones, confiscación de cuentas corrientes y también de ahorros (Planos Collor $\left.{ }^{14}\right)$.

12 Para mayor profundización se puede revisar la Constitución de la República Federativa de Brasil: http://www.planalto.gov.br/ccivil_03/constituicao/constituicao.htm

13 Fuente Archivo Nacional: http://www.arquivonacional.gov.br/cgi/cgilua.exe/sys/start.htm?tpl=home

14 Para mejor comprensión sobre el tema del periódico, revisar la página web Folha de São Paulo: 
LA TRANSFORMACIÓN DE LAS DEMOCRACIAS LATINOAMERICANAS: EL REPLANTEAMIENTO DE LA RELACIÓN ESTADO-SOCIEDAD. CASO BRASIL

Otros casos también ayudaron a debilitar la imagen del presidente. En la mitad del año 90 el presidente fue acusado de corrupción pero esa vez en relación a gastos de su campaña por haber, supuestamente, utilizado agencias publicitarias sin licitación. Collor llegó a procesar el periódico "Folha de São Paulo" ${ }^{15 "}$ por las denuncias que llegaron a ser comprobadas por la justicia brasileña. Posteriormente más escándalos sucedieron cuando el hermano del presidente, Pedro Collor denunció un esquema de corrupción que involucraba el ex tesorero de la campaña del presidente, Paulo César Farias (PC Farias), donde se afirmó ser ilícitas sus riquezas.

Con documentos que incriminaban al tesorero, y que comprobaban siete empresas en el nombre de PC Farias en el exterior, Pedro Collor denuncia a la prensa el fraude de dinero público, lo que presiona al gobierno para investigar las denuncias.

Para aumentar esa presión la Orden de Abogados de Brasil (OAB), la Confederación Nacional de Obispos de Brasil (CNBB), la Central Única de Trabajadores (CUT) y partidos de oposición, realizaron una manifestación conocida como "Vigilia por la ética en la política" para presionar al Congreso Nacional a realizar las investigaciones. Se instaura, entonces, una Comisión Parlamentaria de Inquerito ${ }^{16}$ (CPI) para investigar las denuncias y punir a los involucrados ${ }^{17}$.

El día 30 de julio de 1992, los periódicos "Folha de São Paulo" y “O Estado de São Paulo ${ }^{18 "}$ publicaron editoriales en los cuales pedían la renuncia del presidente. En el día 28 de julio un reportaje de la revista

http://www1.folha.uol.com.br/fsp/dinheiro/fi1603200534.htm

15 Para consulta, periódico Folha de São Paulo: http://www.folha.uol.com.br/

16 (CPI - Comissão Parlamentar de Inquérito) Es un procedimiento realizado por la Cámara o el Senado dependiendo del político a ser juzgado, para investigar posibles irregularidades.

17 Es responsabilidad del Senado Federal procesar y juzgar los casos de crímenes que involucren el presidente de la República. Art. 52. Compete privativamente ao Senado Federal: I - processar e julgar o Presidente e o Vice-Presidente da República nos crimes de responsabilidade, bem como os Ministros de Estado e os Comandantes da Marinha, do Exército e da Aeronáutica nos crimes da mesma natureza conexos com aqueles; http://www.planalto.gov.br/ccivil_03/constituicao/constituicao.htm

18 Para consulta, periódico O Estado de São Paulo: http://www.estadao.com.br/ 
"Isto É"19, presentó un reportaje donde el entonces chofer que trabajaba para el gobierno, Eriberto França, denunciaba un esquema integrado por PC Farias y la secretaria de Collor, Ana Aciólii ${ }^{20}$. Importante destacar aquí que PC Farias fue encontrado muerto junto a su novia Suzana Marcolino en 1996, donde supuestamente cometió suicidio. Un crimen que guarda sus misterios por la coyuntura política del país, donde hasta hoy no se sabe exactamente lo que pasó.

Además de eso los estudiantes empezaron a unirse alrededor de un movimiento estudiantil llamado UNE (Unión Nacional de Estudiantes) que tenía en esa época como líder estudiantil al actual senador Lindberg Farias (PT). Sus reuniones involucraban la movilización de estudiantes de las principales universidades del país, principalmente en las provincias de Río de Janeiro y São Paulo. Todo eso culminó en una protesta que ocurrió el día 11 de agosto de 1937 donde cerca de 10 mil personas protestaron delante del museo de arte contemporáneo (MASP) en São Paulo. Estas protestas están marcadas por la alta participación de estudiantes, por la irreverencia y el apartidismo.

Mirando más a los actores de ese movimiento social que se formó casi que exclusivamente por estudiantes podemos comprender desde una lógica Tourainiana ${ }^{21}$ que la estructura no es determinante de la acción colectiva, sino los actores. Es decir, se rechaza la idea de una masa inconsciente planteada por LeBon ${ }^{22}$, para demonstrar la fuerza de un proceso consciente que como veremos más adelante logrará cambiar la estructura del sistema. La sociedad es dinámica y por eso debe ser vista como un complejo de relaciones, y los movimientos sociales transforman esas relaciones pues son parte de un tejido social con poderes de cambio.

Aquí se logra ver claramente esa masa conscientemente movilizada ma-

19 Para consulta, la revista Isto É: http://www.istoe.com.br/capa

20 Toda esta narrativa fue basada en un reportaje especial sobre el derrocamiento del presidente Collor disponible en la página web del periódico O Estadáo:

http://acervo.estadao.com.br/noticias/topicos,impeachment-de-collor,887,0.htm

21 Alain Touraine, 1987, "Los movimientos sociales, ¿objeto particular o problema central del análisis sociológico?, en El regreso del actor, Buenos Aires: Editorial Universitaria.

22 Gustave LeBon, The Crowd, In Social Movements: A Reader. 
nifestarse en un movimiento llamado de los "caras pintadas" que surgió en respuesta a un pedido de apoyo del presidente Collor. Él hizo un pronunciamiento en la televisión el día 13 de agosto de 1992 convocando a la población a salir a las calles el domingo 16 de agosto de 1992 vistiendo colores nacionales (verde y amarillo). Pero lo que se vio fue justo lo contrario, los estudiantes salieron a las calles vistiendo de negro, con las caras pintadas y gritando "fuera Collor", por eso el nombre de caras pintadas.

Por fin, el movimiento estudiantil a través de reuniones con líderes y mediante propuestas precisas y objetivas lograron lo que buscaban: la renuncia del presidente o su derrocamiento. Post manifestaciones, la Orden de los Abogados de Brasil (OAB) entró con un pedido formal para el derrocamiento del presidente, que fue admitido. Collor fue acusado de enriquecerse con dinero obtenido de manera ilícita por PC Farias y fue considerado culpable de haber recibido 6,5 millones de dólares de manera ilícita.

El proceso fue aprobado con 441 votos a favor y 38 en contra y el presidente fue alejado de sus funciones el 29 de septiembre de 1992. En ese mismo día Collor renuncia pero el proceso corre su curso normal ${ }^{23}$ y su pena fue, además del alejamiento de las funciones, la pérdida de los derechos políticos por ocho años ${ }^{24}$.

De 1992 hasta 1995 permanece en el poder el vice-presidente Itamar Franco. Con el término de su mandato, en las elecciones de 1994 empieza una realidad que se va a repetir durante las elecciones subsecuentes hasta los días actuales: el dualismo partidista en las segundas vueltas presidenciales: PT vs PSDB o la derecha vs la izquierda.

Hagamos una pausa en la historia para explicar el papel que juega el sistema de partidos en Brasil y cómo él puede influir en la relación Es-

23 Basado en un reportaje especial sobre el derrocamiento del presidente Collor disponible en la página web del periódico $\mathrm{O}$ Estadão:

http://acervo.estadao.com.br/noticias/topicos,impeachment-de-collor,887,0.htm

24 Para profundizar en el tema de la campaña presidencial, el papel de la sociedad y medios de comunicación ver Ribeiro, Ilana Aló Cardoso. Medios sociales vs medios de comunicación: la tensión entre la "vieja" y la "nueva" manera de protestar. En: ALACIP - Associação Latino-americana de Ciencias Políticas. Lima- Peru. Anais de eventos, 2015. 
tado-sociedad. Y, para entender el sistema de partidos brasileño primeramente es importante señalar que según el Tribunal Superior Electoral (TSE) existen 32 (treinta y dos) partidos ${ }^{25}$. La fragmentación partidaria se muestra no solamente observando los datos numéricos de los partidos, sino también verificando el número efectivo de partidos en ese sistema. Y, según la fórmula de Markku Laakso y Rein Taagepera, que es una medida internacionalmente aceptada, Brasil posee un número de 11,15 partidos efectivos ${ }^{26}$.

Se señala que como partido político, será utilizada la definición de Alan Ware que dice que "un partido político es aquel que (a) busca influencia en el seno de un Estado, a menudo intentando ocupar posiciones en el gobierno y (b), puesto que normalmente defiende más de un único interés social intenta, hasta cierto punto, "agregar intereses»" (Ware,2004: 31).

Se utiliza esa definición por las diferentes dimensiones que ella trae. Es decir, los partidos políticos son instituciones representativas que por ser institucionalizadas, o sea, parte del sistema, agregan intereses de sus representantes influyendo en el seno de un Estado. Y, por a menudo ocupar posiciones de gobierno, hay una búsqueda por defender intereses sociales colectivos, para detener y mantener el poder.

Y utilizamos la definición de partidos políticos de Duverger, que lo define como la coexistencia de varios partidos en un mismo sistema. Sustenta que "salvo en los Estados con partido único, varios partidos coexisten en un mismo país: las formas y las modalidades de esa coexistencia definen el sistema de partidos del país considerado" (Duverger, 1988: 231).

Así el sistema de partidos brasileño puede ser considerado un sistema fragmentado por la cantidad de partidos existentes aunque debemos tener en cuenta la relevancia o importancia de los partidos en un sistema de partidos. Como propone Sartori el criterio de relevancia no necesariamente se basa en el tamańo del partido, ya que la grandeza o

25 Anexo 2 Fuente TSE: http://www.tse.jus.br/partidos/partidos-politicos/registrados-no-tse/view

26 Fuente de la información página web del PPS (Partido Popular Socialista): http://portal.pps.org. br/portal/showData/257429 
pequeñez de un partido será medida por su fuerza. La fuerza del partido es traducida por su fuerza electoral.

Por lo tanto, la importancia de un partido se mide por: (1) su fuerza electoral: los votos se traducen en escaños y eso nos lleva a la fuerza del partido, (2) potencial de gobierno, que se traduce en: a) potencial de coalición: el partido debe haber sido necesario, al menos en una ocasión, para crear una coalición viable capaz de hacerse con el gobierno, b) potencial de chantaje: la existencia del partido debe afectar a las tácticas de competición de aquellos partidos que tienen potencial de coalición.

Entre las causas de la fragmentación está el sistema de votación. El sistema brasileño es un sistema proporcional, así los partidos poseen más facilidad en elegir diputados en grandes circunscripciones electorales como São Paulo (entre 7 y 70), por ejemplo. Es decir, la "proporcionalidad como principio de decisión significa que el resultado de la elección se decide según la proporción de los votos emitidos obtenida por cada candidato o partido (principio proporcional)" (Nohlen, 1995: 40). Aunque según Nohlen, las fórmulas proporcionales tienden a aumentar la fragmentación partidaria porque los partidos pequeńos tienen posibilidades de obtener escańos en el parlamento.

Pero, aunque Brasil sea un país con una fragmentación partidaria muy grande, son dos los partidos que llegan a la segunda vuelta en las elecciones presidenciales: PSDB, partido que se auto considera de derecha y PT que se auto considera de izquierda ${ }^{27}$. Lo que demuestra que, además de una bipolarización partidaria, existe una bipolarización ideológica que determina también sus coaliciones y alianzas político partidistas $^{28}$.

Eso dice mucho en la relación de la sociedad con el Estado, es un indicador de una sociedad que también se divide en el momento de votar. Y si volvemos al momento histórico que estamos narrando del binomio

27 Fuentes: https://www.pt.org.br/ y http://www.psdb.org.br/

28 Sobre el sistema de partidos brasileńo ver: Ribeiro, Ilana Aló Cardoso. (2015). Sistema de partidos: cuando la corrida electoral se vuelve una guerra. El caso brasileño de las elecciones presidenciales de 2014. Quito - Ecuador. Anais de eventos. In: III Congreso Latinoamericano y Caribeño de Ciencias Sociales. 
PSDB/ PT gana el PSDB con Fernando Henrique Cardoso que va a conducir el país por dos mandatos consecutivos de 1995 hasta 2003.

Su primer mandato (1995-1999) es marcado por implementaciones de políticas neoliberales, la creación de la moneda actual, el real y de la estabilización de la economía, de la política y de la inflación. Viniendo de un mandato conturbado como fue el del presidente Collor, Fernando Henrique logra hacer de sus políticas un periodo de bonanza para el país.

No obstante en su segundo mandato (1999-2003), en donde disputó una vez más la segunda vuelta con el candidato del PT, Lula da Silva, vio llegar la crisis económica mundial que afectó también a Brasil. La solución encontrada fueron las privatizaciones, entre ellas de VALE, creada en la época de Getulio Vargas. Las privatizaciones fueron desde la venta de empresas estatales hasta bancos, carreteras y aeropuertos, medidas consideradas impopulares que generan fricción en la relación Estado-sociedad por el aumento del costo de vida. ${ }^{29}$

En las elecciones siguientes, una vez más en segunda vuelta, se enfrentan PSDB vs PT, de esa vez, José Serra y Lula. El PSDB mantiene desde el segundo mandato presidencial una baja popularidad por las medidas implementadas para intentar salir de la crisis económica, y entonces vence por primera vez, post régimen militar, un partido de izquierda para gobernar el país.

\section{Un giro a la izquierda, un nuevo modelo social y el retorno a la crisis}

El gobierno del PT empieza en 2003 con Luiz Inácio Lula da Silva y permanece hasta los días actuales con Dilma Rousseff. Aquí, la coyuntura juega un papel preponderante, hay un giro a la izquierda en Brasil rumbo a una presentación política diferente a lo que se había implementado anteriormente.

29 Fuente:

http://oglobo.globo.com/economia/privatizacoes-ganharam-forca-partir-dos-anos-90-10448501 
LA TRANSFORMACIÓN DE LAS DEMOCRACIAS LATINOAMERICANAS: EL REPLANTEAMIENTO DE LA RELACión Estado-SOCIEDAD. Caso Brasil

Lula, al contrario de su antecesor, estatiza nuevas empresas, crea universidades federales y promueve concursos públicos, haciendo "crecer" el Estado. En su gobierno, disminuye las divisas externas pero aumenta el endeudamiento interno ${ }^{30}$. Incrementó la generación de empleos, de acuerdo con el IBGE (Instituto Brasileño de Geografía y Estadística), de 2003 hasta 2006 la tasa de desempleo cayó y el empleo formal creció ${ }^{31}$.

Su gobierno también se caracteriza por la creación de políticas de ayuda para las personas consideradas en la línea de la pobreza, además de bajar impuestos y estimular la economía. Creó e incrementó programas sociales donde el objetivo principal estaba en su slogan de gobierno: "Fome zero" (hambre cero). ${ }^{32}$

Bajo ese ideal fue posible la realización de muchos programas sociales pero el mayor de ellos es el "Bolsa Familia". Un programa que busca atender, mediante una ayuda mensual dada por el gobierno, a familias en situación de pobreza y de extrema pobreza ${ }^{33}$. Algunos de los requisitos para recibir el beneficio eran que las familias debían tener renta por persona de $\mathrm{R} \$ 60$ hasta máximo $\mathrm{R} \$ 120$, permanencia escolar de sus niños hasta los quince ańos con asistencia mínima de $85 \%$ y las vacunas actualizadas, entre otros. Ese programa benefició en el año 2008 a 11 millones de familias y su costo para el gobierno fue de 10,9 mil millones de reales ${ }^{34}$.

Otros programas también se destacaron en el gobierno del PT, entre

30 Lula fue citado por el presidente del FMI en una conferencia en 2003 como ejemplo. Fuente: Press Conference with IMF Managing Director Horst Köhler and First Deputy Managing Director Anne Krueger International Monetary Fund Washington, D.C. April 10, 2003. http://www. imf.org/external/np/tr/2003/tr030410.htm

31 Fuente: http://www.ibge.gov.br/home/

32 Fuente: http://www.institutolula.org/projeto-fome-zero-2000-2001/

http://www.mds.gov.br/saladeimprensa/noticias/2011/agosto/Fome,P20Zero,P20Vol1.pdf. pagespeed.ce.qqzWYtqMJC.pdf

33 "O Bolsa Família é um programa de transferência direta de renda que beneficia famílias em situaçáo de pobreza e de extrema pobreza em todo o país. O Bolsa Família integra o Plano Brasil Sem Miséria, que tem como foco de atuação os milhôes de brasileiros com renda familiar per capita inferior a $\mathrm{R} \$ 77$ mensais e está baseado na garantia de renda, inclusão produtiva e no acesso aos serviços públicos." Fuente: http://www.mds.gov.br/bolsafamilia/

34 Fuente: http://politica.estadao.com.br/noticias/geral,veja-os-principais-programas-sociais-dogoverno-lula, 130446 
ellos el "Programa de erradicación del trabajo infantil" que concede una ayuda de hasta $\mathrm{R} \$ 40$ para la familia que promueva la educación del niño que haya estado expuesto al trabajo infantil. Ese programa benefició a 873 mil niños y adolescentes y tuvo un costo para el Estado en 2008 de 368 millones de reales. También el programa "Luz para todos" que busca universalizar el acceso a la luz eléctrica, que tuvo como beneficiarios a 7,2 millones de personas y la inversión fue de 3,5 mil millones de reales.

Otro programa que hay que destacar fue "Brasil alfabetizado" y "Educación para jóvenes y adultos" que busca dar educación básica para jóvenes y adultos contando con la ayuda de los Estados, municipios y universidades. De esto se beneficiaron 8,9 millones de personas y el costo para el Estado en 2008 fue de 381 millones de reales.

Por ultimo vale la pena destacar el PROUNI, un programa que busca llevar la universidad para todos, o sea, permitir el acceso de jóvenes de familias pobres a cursos de tercer nivel a través de becas. Los beneficiarios sobrepasan el número de 116 mil personas. ${ }^{35}$

A través de esos programas, se puede notar claramente el cambio de política del gobierno PSDB respecto al gobierno PT, de un Estado mínimo para un Estado garante. En otras palabras, el Estado ha recuperado espacios de gestión perdidos en los años neoliberales y se proyecta como un actor importante (Acosta, 2012).

El retorno del Estado o el socialismo del siglo XXI tiende a cambiar la relación Estado-sociedad, pues el Estado deja de ser apenas un mediador de las relaciones comerciales a ser participe y garante de las transformaciones sociales. Un Estado agrandado que trabaja en favor de equilibrar las desigualdades sociales mediante políticas asistenciales.

Pero, el gobierno de Lula está marcado por fuertes escándalos de corrupción en su partido (PT) y por la crisis mundial de 2008. Se puede observar el gran gasto estatal para el mantenimiento de sus programas sociales y con la crisis la economía sufrió una fuerte desestabilización ${ }^{36}$.

35 Fuente: http://politica.estadao.com.br/noticias/geral,veja-os-principais-programas-sociais-dogoverno-lula, 130446

36 Medidas del gobierno Lula para estabilizar la economía. Fuente: http://economia.uol.com.br/ ultnot/2008/12/11/ult4294u2001.jhtm 
Fueron muchos escándalos de corrupción revelados por la investigación de la Policía Federal de Brasil. Entre los más destacados están los fraudes en el programa "Bolsa familia" 37 y el "mensaláo" como es conocido el esquema de corrupción donde miembros del partido de los trabajadores recibían soborno por votos en el parlamento. Ese caso llegó al Supremo Tribunal Federal y tuvo políticos condenados por esa corte ${ }^{38}$.

Es con la economía en crisis y el escándalo del "mensalão" que Lula termina su segundo mandato en 2011. Una vez más se repite el duelo entre PSDB con José Serra y PT, ganando el PT con Dilma Rousseff.

La presidenta busca seguir las mismas políticas de su antecesor, pero en un escenario diferente. Las políticas sociales tienen un alto costo de inversión y la crisis económica en la que todavía se encontraba el país hizo incrementar el costo de vida y alzó los impuestos. Todo eso sumado a las inversiones del gobierno para la realización del mundial de futbol en 2013, hizo explotar la relación Estado-sociedad en protestas generalizadas por todo el país.

Todo empezó por el aumento en el pasaje del transporte público a 20 centavos. En el año de 2013, año que el país fue sede de la Copa de las Confederaciones, pre Copa del Mundo que se realizó en 2014 (Mundial 2014), empezaron una serie de movimientos sociales con reivindicaciones distintas que se multiplicaron por todo el país.

Tuvieron inicio en la ciudad de Porto Alegre (sur del país) y Manaus (norte del país) en marzo de 2013 por el aumento en el pasaje mencionado. En mayo el movimiento se extendió para Goiania (centro del país) y en otras ciudades del noreste, hasta llegar a São Paulo (capital más importante del país ubicada en el sureste). Aquí surge un actor protagónico, el Movimiento Pase Libre (MPL), que a partir de un anuncio

37 Fuente: http://www.theguardian.com/global-development/2013/dec/19/brazil-bolsa-familiapolitical-tool-social-welfare

http://veja.abril.com.br/blog/reinaldo/geral/lula-o-bolsa-familia-os-detalhes-de-uma-farsa-euma-falha-escandalosa-da-imprensa/

38 Fuentes: http://g1.globo.com/politica/mensalao/index.html http://www1.folha.uol.com.br/especial/2012/ojulgamentodomensalao/ http://www.economist.com/blogs/economist-explains/2013/11/economist-explains-14 http://www.bbc.com/news/world-latin-america-19081519 
de aumento del precio del pasaje de bus resolvió organizar manifestaciones en contra del aumento.

Lo mismo pasó con protestas en la ciudad de Río de Janeiro (una de las principales capitales del país ubicada en el sureste). Inicialmente todas esas protestas fueron por una misma cuestión, el aumento del costo del pasaje en los medios de transporte. De acuerdo con el crecimiento y la intensificación de las protestas la respuesta del gobierno fue cada vez más represiva y el escenario se fue modificando poco a poco. Se agregó a ese movimiento otros movimientos y reivindicaciones mientras que las protestas se volvieron más violentas y susceptibles a infiltración de personas enmascaradas que cometían crímenes y vandalismo.

Se destaca que los movimientos sociales que salieron a protestar no logran ser captados por partidos porque esos no supieron responder de manera eficiente a la sociedad, por eso una característica que marcó esas manifestaciones también fue el apartidismo.

Las protestas seguían de manera desenfrenada con alta participación popular y una insatisfacción generalizada que llevó a confrontaciones graves entre manifestantes y policiales con algunos muertos. A medida en que se aproximaba la Copa de las Confederaciones las protestas también aumentaron así como las reivindicaciones. Se agregaron al Movimiento Pase Libre, la cuestión de la PEC 37 (Proyecto de enmienda constitucional para luchar contra la impunidad), la PEC 33 (Proyecto de enmienda constitucional sobre actos médicos), el aumento del costo de vida, el volumen de gastos públicos por la realización de la Copa de 2014 y las olimpiadas de 2016 en Río de Janeiro, una mayor inversión estatal en salud y educación, entre otras reivindicaciones.

Es posible distinguir tres fases de la protesta: la primera que se enfocó en las tarifas de transporte, la segunda con fuerte apoyo popular pero dispersa en muchas reivindicaciones y la tercera marcada por actos de vandalismo, una retirada de la población en general para que se queden los adeptos a la táctica de los black blocks. Esta táctica consiste en un grupo de personas que se visten de negro para parecer una sola masa y 
atacan símbolos de poder y del capitalismo ${ }^{39}$.

El logro en la estructura de oportunidades políticas vino después de que la manifestación llegó a Brasilia (capital del país) el 20 de junio de 2013. Primeramente, la respuesta vino a nivel regional. Los alcaldes de las ciudades que se manifestaron contra el aumento del pasaje de los medios de transporte volvieron a la tarifa anterior. Las protestas fueron reprimidas con fuerte violencia policial y eso fue detenido por la ciudadanía, así que tanto a nivel regional como nacional, las propuestas se centraron en una baja de la represión policial. La presidenta, por su parte, sin saber muy bien qué atender propuso cinco ejes centrales para que cesen las manifestaciones en el país a través de un pronunciamiento en la televisión nacional el día 21 de julio de 2013.

La propuesta se centró en cinco pactos y la posibilidad de un plebiscito para la reforma política: (1) La cuestión del trasporte público (mejoría en los servicios y revisión de tarifas); (2) Reforma política y combate a la corrupción (plebiscito); (3) Inversión en la salud (convocatoria nacional e internacional, programa "Más médicos"); (4) Inversión en educación (regalías del petróleo y pre sal); (5) Responsabilidad fiscal (control de la inflación y estabilidad económica) ${ }^{40}$.

Con el fin de las protestas y del Mundial 2014, Dilma pudo preocuparse por su reelección. La corrida presidencial de 2014 fue marcada por la disputa habitual del PT (Dilma Rousseff) y PSDB (Aécio Neves) pero con un nuevo actor que por poco termina con ese binomio de años, el PSB (Marina Silva).

Hubo una polarización del electorado en dirección a esos candidatos y el resultado de la primera vuelta fue: en primer lugar Dilma Rousseff (PT) con 41,59\% de los votos válidos; en segundo lugar Aécio Neves (PSDB) con 33,55\% de los votos válidos y en tercer lugar Marina Silva (PSB) con 21,32\% de los votos válidos ${ }^{41}$.

39 Reportaje Folha de São Paulo de 27/12/2013: http://www1.folha.uol.com.br/poder/ 2013/12/1390207-manifestacoes-nao-foram-pelos-20-centavos.shtml

40 Toda la narrativa fue basada en un especial del sitio web G1 de la red Globo sobre las manifestaciones del 2013: http://g1.globo.com/brasil/linha-tempo-manifestacoes-2013/platb/

41 Estadística presidencial 2014. Fuente TSE http://www.tse.jus.br/eleicoes/estatisticas/estatisticas- 
Lo que sucede después del resultado electoral es una lucha para realizar alianzas, coaliciones y centralizar apoyos para intentar conquistar los electores de los partidos no vencedores. Aquí cabe hablar de la coherencia partidista, principalmente en cuanto al apoyo de la candidata Marina Silva (PSB) que lo manifestó a favor del candidato Aécio Neves, lo que ocasionó muchas repercusiones ${ }^{42}$.

Se puede destacar, por ejemplo, el impacto que esa decisión produjo en su propio partido PSB, que en una clara demonstración de incoherencia partidista, principalmente ideológica (porque se trata de un partido de izquierda), su decisión promovió una cisura en ese partido.

Si partimos de la máxima de que los partidos políticos representan parcelas de la sociedad, podemos entender que las elecciones de 2014 reflejan el momento social vivido en el país y la relación que imprime el Estado con la sociedad. Dilma Rousseff es electa con 51,64\% de los votos válidos en contra de su opositor Aécio Neves con 48,36\% de los votos válidos ${ }^{43}$.

Una reelección complicada y un inicio de segundo mandato marcado también por la inestabilidad política. Brasil sigue hundido en una crisis económica que sigue aumentando el costo de vida y llevando el país a un estado de recesión que se refleja en la disminución de su PIB ${ }^{44}$.

Con la situación económica difícil y la explosión del escándalo de corrupción de la estatal brasileña de petróleo Petrobras, que ayudó a generar aún más crisis económica, el actual gobierno enfrenta su más grave crisis ${ }^{45}$. La presidenta goza de una baja popularidad y en el país

eleitorais-2014-resultado

42 Fuente: http://g1.globo.com/politica/eleicoes/2014/coligacoes-partidarias/infografico/index.html

43 Fuente: http://eleicoes.uol.com.br/2014/noticias/2014/10/26/dilma-cresce-na-reta-final-ereeleita-e-emplaca-quarto-mandato-do-pt.htm

44 Fuentes: http://www.gazetadopovo.com.br/economia/brasil-a-beira-da-recessao-economicaebo881khyovchk3o8tbjcmvym

http://g1.globo.com/economia/noticia/2015/08/moodys-tem-previsao-de-recessao-nobrasil-em-2016.html

http://g1.globo.com/economia/noticia/2015/08/economia-recua-19-no-2-trimestre-de-2015. html

45 Escándalo de corrupción Petrobrás. Fuentes: http://g1.globo.com/bom-dia-brasil/noticia/ 2015/07/pf-calcula-que-prejuizo-da-corrupcao-na-petrobras-pode-chegar-r-19-bi.html http://wwwl.folha.uol.com.br/especial/2014/petrolao/ http://www1.folha.uol.com.br/infograficos/2014/09/114361-escandalo-na-petrobras.shtml 
ocurren cada vez más y más protestas friccionando la relación del Estado con la sociedad por el aumento de la insatisfacción de muchos sectores. Los medios de comunicación y las encuestas demuestran que es la más baja popularidad desde el gobierno de Collor de Melo, con un $71 \%$ de reprobación ${ }^{46}$. Queda saber si la historia se repite aunque en escenarios diferentes.

\section{Consideraciones finales}

Hacer un análisis histórico y político de la República brasileña desde sus inicios nos da la capacidad de rever el pasado con vistas al futuro. Este breve artículo buscó como su objetivo principal abordar las transformaciones de la democracia brasileña para responder a la pregunta de investigación que gira alrededor de la relación Estado-sociedad.

La breve historia Republicana está marcada por mucha represión y una relación altamente vertical entre esos dos polos. Y, para que vuelva la democracia tuvo que haber una gran aclamación popular que culminó con el fin del régimen militar. El pueblo empieza entonces a redescubrirse, redescubrir el poder que reside en manifestarse, el poder que tiene para elegir un presidente y derrocarlo, o sea, su poder original como pueblo: el pueblo que detiene el poder en la democracia y es capaz de buscar un replanteamiento en como relacionarse con el Estado.

Pues delante de todo eso, ¿es posible decir que desde el retorno a la democracia hubo un replanteamiento de la relación Estado-sociedad? La conclusión es que sí. Esa relación parte en una creciente donde la verticalidad se vuelve cada vez más horizontal. En otras palabras, según la historia, el Estado vertical y represor de la dictadura, con el regreso a la democracia va pasar a ser un regulador hasta llegar a una posición de

46 Fuentes sobre la popularidad: http://www1.folha.uol.com.br/poder/2015/08/1665135reprovacao-de-dilma-cresce-e-supera-a-de-collor-em-1992.shtml http://g1.globo.com/politica/noticia/2015/08/71-reprovam-governo-dilma-diz-datafolha.html Fuente sobre las protestas: http://g1.globo.com/politica/noticia/2015/08/manifestantesprotestam-contra-o-governo-dilma-em-cidades-do-brasil.html 
garante y actor protagónico generador de políticas.

Todo eso es posible por las diferentes maneras de hacer política que hacen que se pueda identificar un cambio en la manera en que el Estado se relaciona con la sociedad y viceversa. El replantear esa relación parte también de una cuestión participativa donde el brasileño aprende a exigir sus derechos utilizando medios no institucionalizados, como la protesta.

Por lo tanto, la relación Estado-sociedad se vuelve cada vez más mutante y se puede inferir que muchos factores influyen para ello. La cuestión económica, política y social siempre va a ser relevante cuando se vaya a analizar esa relación, así como la coyuntura. El pasado se refleja en el futuro donde el cambio constante construye nuevas historias.

\section{Bibliografía}

Acosta, A. (2012). El retorno del Estado. Primeros pasos postneoliberales, más no postcapitalistas. Quito. La Tendencia No 13, abril-mayo.

Duverger, M. (1988). Los partidos políticos. México. Fondo de Cultura Económica: [primera edición: 1951].

Harvey, D. (2013). Breve historia del neoliberalismo. Madrid: Akal. Hirst, M. (s/a). “La época de Vargas: 1930/1945”, en Crítica y Utopía $\mathrm{N}^{\circ} 5$.

Huntington, S. (1989). "El sobrio significado de la democracia". Santiago: Revista de Estudios Públicos No33.

Huntington, S. (1994). La tercera ola. La democratización a finales del siglo XX. Argentina: Editorial Paidós.

Laclau, E. (2013). A Razão Populista. Rio de Janeiro: EdUERJ.

LeBon, G. (s/a). The Crowd in Social Movements: A Reader.

Montahuano, F. (2015). "Modificaciones a la flexibilización laboral: tramas del post-neoliberalismo en el Ecuador". Quito - Ecuador. En: III Congreso Latinoamericano y Caribeño de Ciencias Sociales. Anais de evento. 
Nun, J. (1995). La democracia y la modernización, treinta años después. Perú: Instituciones Políticas y Sociedad IEP.

Ribeiro, Ilana Aló Cardoso (2015) Medios sociales vs medios de comunicación: la tensión entre la "vieja" y la "nueva" manera de protestar. Lima- Perú. Anais de eventos. ALACIP - Associação Latino-americana de Ciencias Políticas.

Ribeiro, Ilana Aló Cardoso (2015). Sistema de partidos: cuando la corrida electoral se vuelve una guerra. El caso brasileño de las elecciones presidenciales de 2014. Quito - Ecuador. Anais de eventos. III Congreso Latinoamericano y Caribeńo de Ciencias Sociales.

Ruiz-Peinado, José Luis (2003). El Brasil de los populismos: de la construcción de la identidad nacional a la supuesta democracia racial. Barcelona: Dossier Universidad Autónoma de Barcelona.

Sartori, Giovanni (1992). Partidos y sistemas de partidos. Madrid: Alianza Editorial.

Touraine, Alain (1987). "Los movimientos sociales, ¿objeto particular o problema central del análisis sociológico?” En "El regreso del actor". Buenos Aires: Editorial Universitaria.

Ware, Alan (2004). Partidos politicos y sistemas de partidos. Madrid: Istmo. 